\title{
Feasibility of an exercise intervention for women with postnatal depression: a pilot randomised controlled trial
}

\author{
Amanda J Daley, Heather Winter, Chloe Grimmett, \\ Mary McGuinness, Richard McManus and Christine MacArthur
}

\begin{abstract}
Background

Postnatal depression is a serious mental health problem that may be reduced by exercise.

Aim

This study examined the feasibility of an exercise intervention for women with postnatal depression, and assessed which methods of recruitment are most effective.

Design of study

Randomised controlled trial.

Setting

General practice and the community

Method

Participants were recruited from various sources and randomised to an exercise intervention or usual care with follow-up at 12 weeks. As well as assessing feasibility, other trial outcomes included exercise participation and self-efficacy for exercise. Levels of depression were assessed but the study was not powered to show a difference in this.

Results

The recruitment rate of eligible patients was $23.1 \%$. The highest recruitment rate was via referral from the psychiatric mother and baby unit $(9 / 28 ; 32.1 \%)$, followed by invitation letters from GPs (24/93; 25.8\%). Thirty-eight eligible participants were randomised. At follow-up there was no significant difference in exercise participation between groups. The intervention group reported significantly higher selfefficacy for exercise compared to usual care, but depression scores did not differ.

\section{Conclusion}

Exercise participation over the 12-week period was not significantly increased, possibly because it is difficult to motivate women with postnatal depression to exercise, or the intervention was not sufficiently intensive. Eligible patients were recruited into this study but response rates were low. Optimum methods of recruitment in this difficult-to-reach population are required prior to a substantive trial. Further research is imperative given poorly-evidenced recommendations by the National Institute for Health and Clinical Excellence to consider this treatment.
\end{abstract}

Keywords

exercise; postnatal depression; women.

\section{INTRODUCTION}

Postnatal depression is a serious mental health problem, ${ }^{1}$ affecting around one in 10 women at some time in the first year., ${ }^{2,3}$ As well as the morbidity suffered by the mother, studies have found an association between postnatal depression and adverse effects on the child. ${ }^{4,5}$ Consideration of novel adjunctive interventions for the treatment of postnatal depression is required, given that some women are reluctant to take antidepressants after giving birth, ${ }^{6}$ and there is limited availability of psychological therapies. Meta-analyses have concluded that exercise may be effective in reducing depression in general populations. ${ }^{7,8} \mathrm{~A}$ recent review identified two small randomised controlled trials which examined the effects of exercise on postnatal depression; ${ }^{9-11}$ both trials reported substantial reductions in postnatal depression scores in participants randomised to receive interventions involving pram pushing. However, the very small samples in these

AJ Daley, PhD, C Psychol, lecturer in health psychology; R McManus, FRCGP, PhD, clinical senior lecturer in primary care, Department of Primary Care and General Practice, University of Birmingham. $\mathrm{H}$ Winter, $M R C O G, M D, F P H$, senior clinical lecturer in public health and epidemiology. C MacArthur, PhD, professor of maternal health and child epidemiology, Department of Public Health and Epidemiology, University of Birmingham. C Grimmett, BSc, research associate, Cancer Research UK Health Behaviour Unit, Department of Epidemiology and Public Health, University College London. M McGuinness, MSc, consultant clinical psychologist, Perinatal Mental Health Service, Queen Elizabeth Psychiatric Hospital, Birmingham.

Address for correspondence

Dr Amanda Daley, The Department of Primary Care and General Practice, Clinical Sciences Building, University of Birmingham, Birmingham B15 2TT.

E-mail: a.daley@bham.ac.uk

Submitted: 11 May 2007; Editor's response: 2 August 2007; final acceptance: 30 October 2007.

(B) British Journal of General Practice 2008; 58: 178-183.

DOI: 10.3399/bjgp08X277195 
studies limit their validity. Both trials, by the same team, were conducted in the Gold Coast, Australia where the year-round warm weather conditions are likely to have positively influenced mothers' decisions to take part; ${ }^{10,11}$ thus contextual factors decrease the applicability of these findings to other settings.

Despite the limited evidence in postnatal women, ${ }^{9}$ the National Institute for Health and Clinical Excellence (NICE) recently recommended in their antenatal and postnatal mental health guidelines that health professionals should consider exercise as a management strategy in women experiencing mildto-moderate depression. ${ }^{12}$ Further research into this issue is required in representative settings.

Limited information has been provided by previous study reports about how participants were accessed or recruited. Prior to any substantive trial, it is important to consider the merits of different methods of identifying cases. Once eligible patients have been recruited to an exercise programme, the demands of a new baby may then make regular exercise participation difficult. Therefore, this study investigated the feasibility and acceptability of an exercise intervention in women with postnatal depression.

\section{METHOD}

\section{Participant eligibility}

Women aged 16 years or more who were experiencing depression, and whose youngest child was less than 12 months' old were eligible. Participants could continue with prescribed medications and any counselling/behavioural treatments, but had to speak English because of lack of interpretation services. Women with severe postnatal depression who required inpatient psychiatric treatment, had psychotic symptoms, or were known to be pregnant were excluded.

A further eligibility check of these criteria was made by the researchers prior to consent, as well as screening for current activity to exclude women who were already active (defined as exercise three or more times per week for at least 30 minutes per session, for more than 3 months) or those who were unable to provide written informed consent (Figure 1).

\section{Recruitment}

To assess the feasibility of a variety of recruitment strategies, cases were identified through four different routes. All general practices within four primary care trusts in Birmingham were informed by letter about the aims of the study. They were invited to assist with recruitment by identifying potentially eligible patients according to trial inclusion criteria. The local specialist mother and baby unit was asked to do the same. General practices and the mother and baby unit then

\section{How this fits in}

Postnatal depression is a serious and common mental health problem.

Evidence from depressed general populations has shown that exercise may be

effective in reducing depressive symptomatology, but there is inadequate

evidence on this in relation to women with postnatal depression. The current

study investigated the feasibility and acceptability of an exercise intervention in

this population. It illustrates that postnatal women are difficult to access and

recruit. The intervention was successful in increasing self-efficacy for exercise,

but exercise participation and levels of depression did not differ at follow-up.

sent invitation letters to eligible women and asked them to contact the trial team if they were interested in participating. Presentations about the trial were made to health visitors in each of the primary care trusts, and they were asked to refer eligible women. Support groups were visited and asked to inform women about the trial so they could self-refer.

Depression was identified using clinical judgement by GPs, psychiatrists, and health visitors, but women recruited by self-referral were screened and had to have a score $>12$ on the Edinburgh Postnatal Depression Scale (EPDS) ${ }^{13}$ to be eligible.

\section{Randomisation}

After the completion of their baseline assessment, participants were randomised to the exercise intervention group or usual care, according to a computer-generated random list provided by an independent statistician.

\section{Outcomes}

Information relating to each method of recruitment was systematically documented. The Godin LeisureTime Exercise Questionnaire was used to assess participants' levels of involvement in mild, moderate, and vigorous exercise. ${ }^{14} \mathrm{~A}$ self-efficacy for exercise questionnaire was used to assess participants' confidence in being active in different situations (for example, when tired, in a bad mood, or when lacking time)..$^{15}$ Members of the exercise group were asked to complete a checklist exercise diary to record the amount of time in minutes of exercise they achieved each day. Levels of depression using the EPDS were obtained, ${ }^{13}$ but the study was not powered to show a difference in this outcome.

\section{Additional information}

Demographic data, current medication, and items relating to participants' health behaviours, including breastfeeding, were collected. If participants were receiving counselling or taking any prescribed medication this was documented, including data regarding participants' exercise history. A social 


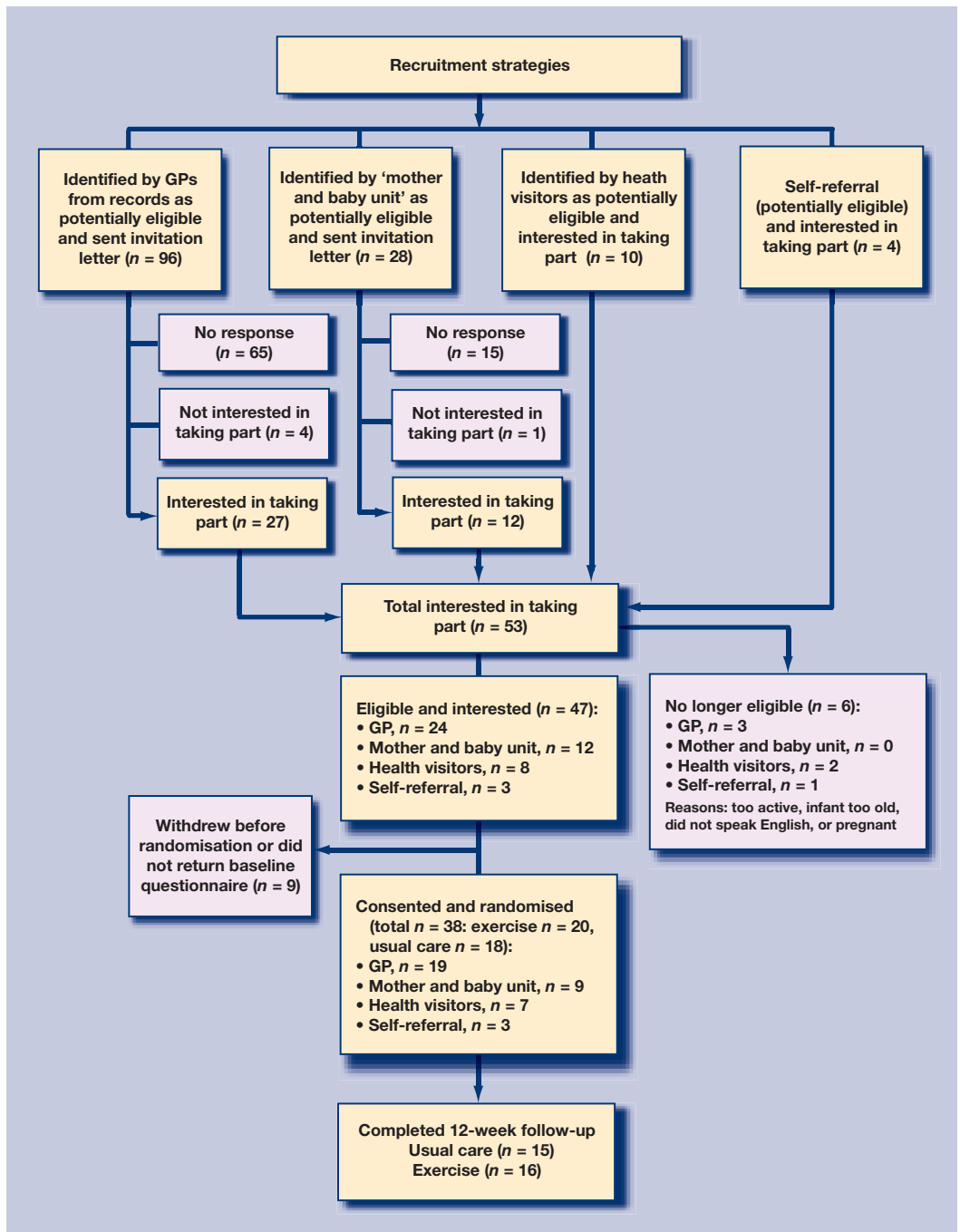

support score was calculated based on women's responses to five items: the amount of help they receive from their partner; the availability of a friend or relative to contact if needed; a local contact who has an infant; the availability of practical help at home; and the duration of residence in the locality. ${ }^{16}$

Those randomised to the exercise intervention completed a series of open-ended questions about their experiences during the intervention. Participants were offered the opportunity to return these evaluations anonymously.

\section{Assessment of outcomes}

All outcomes were assessed at baseline and 12 weeks after the end of exercise intervention using the postal questionnaire at each time point, and with reminders sent to non-responders after 2 weeks.

\section{Exercise intervention}

Participants in the intervention group were offered two one-to-one exercise consultations over the 12week intervention period. Further information regarding the theoretical basis of the intervention and the intervention approach used can be found elsewhere. ${ }^{17}$ The intervention aimed to equip individuals with the skills, knowledge, and confidence needed to participate in regular exercise, and was delivered in participants' homes by a trained researcher. Consultations lasted about 1 hour.

The first consultation focused on uptake of exercise and enhancing motivation, self-efficacy for exercise, overcoming barriers, and developing appropriate activity goals. Participants were also given a pedometer. A short 'walk and talk' prampushing session was incorporated within the first consultation so that practical issues, such as perceived exertion monitoring and exercise safety, could be explored with participants.

Four weeks later, a second consultation focused on the prevention of relapse back to sedentary behaviour and/or improving maintenance of an active lifestyle. Follow-up support phone calls lasting about 10 minutes were given during weeks 3 and 9, where any persistent barriers or issues preventing behavioural change were discussed.

The behavioural goal was for women to work towards accumulating participation in moderateintensity activities for at least $\mathbf{3 0}$ minutes per day, five days of the week. Walking in the form of 'pram pushing' was advocated and, when appropriate, opportunities for women to exercise without their baby were discussed.

\section{Usual care}

Members of the usual care group were asked not to change their current exercise patterns but were offered an exercise consultation at the end of their involvement in the study.

\section{Sample size and analysis plan}

The primary outcome was the frequency of moderateintensity exercise per week. The Department of Health recommends at least 30 minutes of moderateintensity physical activity per day for at least 5 days per week (minimum total of 150 minutes per week) for the general population. ${ }^{18}$ This study expected women to achieve $70 \%$ (105 minutes) of this target, and the sample was sufficient to estimate weekly exercise with $14 \%$ precision ( $95 \%$ confidence level). Analyses of covariance (controlling for baseline scores) were used to compare outcomes between groups at 12-weeks' follow-up. Data were analysed on an intention-to-treat basis.

\section{RESULTS}

\section{Recruitment of general practices}

Of 262 practices invited to assist with study recruitment, 56 (21\%) agreed, 67 (26\%) declined, and 
139 (53.1\%) did not respond. Of those practices that agreed $(n=56)$, three later withdrew, 18 provided no patient lists when requested, and 10 reported no eligible patients at the time of the study; thus $25 / 262$ $(9.5 \%)$ practices provided lists of eligible patients.

\section{Patient recruitment}

Recruitment took place between October 2005 and May 2006. Fifty-three women expressed an interest in taking part, of whom 27 (51\%) were identified from general practice records, $12(23 \%)$ by the specialist mother and baby unit, 10 (19\%) from health visitor referral, and four (8\%) self-referred. A higher proportion of women responded to an invitation from the mother and baby unit $(12 / 28 ; 42.9 \%)$ than their GP (27/96; 28.1\%). Six women were subsequently found to be ineligible, and nine withdrew prior to randomisation or did not return the baseline questionnaire, leaving 38 eligible patients who were randomised (recruitment rate 4-5 patients per month). Denominator data were only available for GPs $(n=96)$ and the mother and baby unit $(n=28)$. Trial recruitment from these sources was $23.1 \%$ (28/121; 124 potentially eligible minus three screened as further ineligible; Figure 1).

\section{Characteristics of randomised participants}

Most participants were aged between 29 and 31 years, had one or two children, lived with their husband/partner, and were not currently breastfeeding their infant. Most participants were employed, taking antidepressants, had low levels of social support, and lived in more deprived communities (Table 1). A total of $26.3 \%$ of the sample were of non-white ethnicity. At baseline, 33 of the 38 participants (exercise group, $n=17$; usual care, $n=$ 16) recorded an EPDS score $>12$, and in 4 of the 38 participants the EPDS score was 12 (exercise group, $n=2$; usual care, $n=2$ ).

\section{Exercise participation and EPDS score}

No significant mean difference between the groups was found for the frequency of participation in mild, moderate, or vigorous exercise, although scores for moderate-intensity exercise favoured the exercise group. The exercise group reported significantly higher adjusted mean self-efficacy for exercise scores than usual care $(P=0.002)$. EPDS scores did not differ significantly between the groups (Table 2).

\section{Adherence (intervention group only)}

Descriptive analysis of data from the exercise diaries of the 11 participants who completed and returned these, indicated that participants engaged in exercise for a mean of 174 minutes (standard deviation $=73.6$ ) per week. Ten of these 11 women
Table 1. Baseline demographic characteristics of participants by study group.

\begin{tabular}{|c|c|c|}
\hline Characteristic & Usual care $(n=18), n(\%)$ & Exercise $(n=20), n(\%)$ \\
\hline \multicolumn{3}{|l|}{ Age, years } \\
\hline $21-30$ & $10(55.5)$ & $10(50.0)$ \\
\hline $31-40$ & $8(44.5)$ & $9(45.0)$ \\
\hline$>40$ & 0 & $1(5.0)$ \\
\hline \multicolumn{3}{|l|}{ Body mass index } \\
\hline Under/normal weight ( $\leq 25)$ & $11(61.1)$ & $10(50.0)$ \\
\hline Overweight $(>25$ to $\leq 30)$ & $4(22.2)$ & $7(35.0)$ \\
\hline Obese $(>30$ to $\leq 40)$ & $1(5.6)$ & $3(15.0)$ \\
\hline \multicolumn{3}{|l|}{ Ethnicity } \\
\hline White & $12(66.6)$ & $16(80)$ \\
\hline \multicolumn{3}{|l|}{ Parity } \\
\hline $1-2$ & 12 (66.6) & $16(80)$ \\
\hline $3-4$ & $6(33.3)$ & $4(20)$ \\
\hline \multicolumn{3}{|l|}{ Index of Multiple Deprivation } \\
\hline Quartile 1 - least deprived & $1(5.5)$ & $1(5.0)$ \\
\hline Quartile 2 & $1(5.5)$ & $1(5.0)$ \\
\hline Quartile 3 & $7(38.9)$ & $4(20.0)$ \\
\hline Quartile 4 - most deprived & $8(44.4)$ & $12(60.0)$ \\
\hline Married/with partner & $16(88.9)$ & $13(65.0)$ \\
\hline \multicolumn{3}{|l|}{ Employment status } \\
\hline Paid employment & $11(61.1)$ & $15(75.0)$ \\
\hline Unemployed & $3(16.7)$ & $1(5.0)$ \\
\hline Looking after family/home & $3(16.7)$ & $3(15.0)$ \\
\hline Sick disabled & 0 & $1(5.0)$ \\
\hline Other & $1(5.5)$ & 0 \\
\hline \multicolumn{3}{|l|}{ Mode of delivery } \\
\hline Normal vaginal delivery & $10(55.6)$ & $12(60.0)$ \\
\hline Instrumental vaginal delivery & $4(22.2)$ & $4(20.0)$ \\
\hline Caesarean section & $4(22.2)$ & $4(20.0)$ \\
\hline Currently breastfeeding & $1(5.5)$ & $5(25.0)$ \\
\hline Taking antidepressants & $9(50.0)$ & $13(65.0)$ \\
\hline \multicolumn{3}{|l|}{ Receiving psychological support } \\
\hline Yes & $9(50.0)$ & $6(30.0)$ \\
\hline No & 7 (38.9) & $9(45.0)$ \\
\hline On waiting list & $1(5.5)$ & $4(20.0)$ \\
\hline \multicolumn{3}{|c|}{ Social support score ${ }^{a}$ (range $=0-15$ ) } \\
\hline$\leq 12$ & $11(61.1)$ & $16(80.0)$ \\
\hline $13-14$ & $7(38.9)$ & $1(5.0)$ \\
\hline 15 & 0 & 0 \\
\hline Smokers & $4(22.2)$ & $4(20.0)$ \\
\hline Physically active prior to pregn & $10(55.6)$ & $7(35.0)$ \\
\hline
\end{tabular}

Participant numbers vary due to missing values. ${ }^{a}$ Higher score represents greater social support.

were able to achieve the intervention goal of at least 105 minutes' exercise per week.

\section{Open-ended questions}

Of women who were randomised to the exercise intervention, 14 (70\%) returned their responses to the intervention evaluation questionnaire. All responders stated exercise should be encouraged in women who are experiencing low mood. Eleven of the 14 women thought that exercise helped to improve their low mood. Twelve women reported that their general health had improved. 
Table 2. Trial outcome data.

\begin{tabular}{|c|c|c|c|}
\hline & $\begin{array}{l}\text { Usual care, } \\
\text { mean (SD) }\end{array}$ & $\begin{array}{l}\text { Exercise, } \\
\text { mean (SD) }\end{array}$ & $\begin{array}{c}\text { Mean difference } \\
(95 \% \mathrm{Cl})\end{array}$ \\
\hline \multicolumn{4}{|c|}{ Mild-intensity exercise ${ }^{a}$} \\
\hline Baseline & $2.3(2.0)$ & $1.9(1.4)$ & 0.7 (-0.8 to 2.3$)$ \\
\hline Follow-up ${ }^{c}$ & 2.4 (1.9) & $3.1(2.0)$ & \\
\hline \multicolumn{4}{|c|}{ Moderate-intensity exercise ${ }^{a}$} \\
\hline Baseline & $0.8(1.4)$ & $1.8(2.9)$ & $1.6(-0.8$ to 4.0$)$ \\
\hline Follow-up ${ }^{c}$ & $1.8(3.0)$ & 3.4 (3.2) & \\
\hline \multicolumn{4}{|c|}{ Vigorous-intensity exercise } \\
\hline Baseline & $0.6(1.0)$ & $0.3(0.8)$ & $0.06(-0.9$ to 0.7$)$ \\
\hline Follow-up ${ }^{c}$ & $0.7(1.2)$ & $0.6(1.2)$ & \\
\hline \multicolumn{4}{|c|}{ Self-efficacy for exercise ${ }^{a, b}$} \\
\hline Baseline & $11.3(3.8)$ & $10.5(3.2)$ & 4.1 (1.6 to 6.4$)$ \\
\hline Follow-up ${ }^{c}$ & $9.5(3.1)$ & $13.6(3.2)$ & \\
\hline \multicolumn{4}{|c|}{ Postnatal depression (EPDS) } \\
\hline Baseline & $19.2(4.7)$ & $17.7(5.2)$ & $1.2(-5.2$ to 2.8$)$ \\
\hline Follow-up ${ }^{c}$ & $14.3(5.4)$ & $13.1(5.2)$ & \\
\hline
\end{tabular}

amean times per week. Mild-intensity exercise refers to activities such as yoga, golf, and easy walking. Moderate-intensity exercise refers to activities such as fast walking, tennis, cycling, and dancing. Vigorous exercise refers to activities such as jogging and aerobics. ${ }^{6}$ Denotes significant difference between groups at $\mathrm{P}<0.05$. ${ }^{\circ} \mathrm{Folllow}$-up scores are adjusted for baseline. Note: follow-up data: usual care, $\mathrm{n}=15$; exercise, $\mathrm{n}=16$. $E P D S=$ Edinburgh Postnatal Depression Scale.
The most popular reasons given for participation in the study were to help depression/low mood $(n=7)$, enhance motivation to exercise $(n=4)$, help others experiencing similar symptoms $(n=3)$, and to have a goal to reach each day and to gain a sense of achievement $(n=2)$.

Most women reported they had enjoyed the exercise they had been doing during the intervention $(n=11)$. The most popular type of exercise was walking. The majority $(n=8)$ felt that they would do more exercise if there were group walking sessions with other women experiencing low mood after childbirth; four women were unsure; and two women did not feel groups would be useful. The main barriers to exercise faced by mothers were childcare and family responsibilities $(n=10)$, lack of time $(n=$ $6)$, feeling tired $(n=4)$, weather $(n=4)$, and physical and psychological health problems related to mother or infant $(n=3)$.

\section{DISCUSSION}

\section{Summary of main findings and comparison with existing literature}

As anticipated, women with postnatal depression proved difficult to access. Responses to the study from general practices was low: only $9.5 \%$ of those approached responded and were able or willing to write to their eligible patients to invite them to take part; therefore, many women who may have been eligible were not given the opportunity to participate. The trial recruitment rate from among eligible patients contacted was disappointing (about 23\%). This might indicate limited interest in exercise as a therapeutic intervention in women experiencing postnatal depression, or that the recruitment strategies were not as effective as they might have been.

The recruitment rate from the mother and baby unit was the most successful, which was encouraging. Women recruited from the mother and baby unit are likely to be have been more depressed, as more severe cases are referred and accepted for treatment by this service. Therefore, they might have been expected to have been more difficult to recruit than those identified from other routes.

The difficulties experienced in accessing eligible patients further emphasizes recent concerns about the difficulties of identifying women with postnatal depression ${ }^{19}$ for appropriate treatment to be offered and to diminish potential harm to the infant. ${ }^{4-5}$

No significant difference was found in the amount of exercise achieved between the groups, although the direction of findings favoured the exercise group. Interestingly, of the women who returned their exercise diary, all except one were able to achieve the intervention goal of at least 105 minutes of exercise per week. The lack of significant differences in exercise outcomes at follow-up may be the result of intervention contamination with trial information, possibly resulting in increased exercise participation in the usual care group, although exercise scores relative to baseline did not generally support this. The intervention applied may not have been adequate to induce an increase in exercise participation for this population of women.

The intervention group reported significantly higher self-efficacy for exercise scores, suggesting the intervention was successful in promoting participants to believe that they could achieve regular participation. Self-efficacy may be a proximal predictor of behaviour, which would impact on mood. Unlike previous research, ${ }^{10,11}$ no significant difference in EPDS scores was found between the groups, but the ability of the present study to detect a significant group difference in EPDS scores would only have been possible if the effect size had been as large as those found in the two previous trials.

The evaluation questionnaire indicated that participants felt they had experienced some mental health benefits, even though EPDS measurement of postnatal depression did not show a significant difference between the groups. Most participants reported they enjoyed the intervention and thought that exercise should be an activity that is endorsed for postnatal women with low mood. The majority of participants felt they would achieve more exercise if group sessions with other women experiencing low mood after childbirth were available, and this should 
be considered in the development of any subsequent interventions.

\section{Strengths and limitations of the study}

In this study, a range of recruitment strategies were assessed and data were provided on which methods are likely to be most effective. The small sample limits the generalisability of the trial findings, but the sample size is larger than both previous trials..$^{10,11}$ The low level of attrition across follow-ups was encouraging and this is the first published study to take place in the UK.

\section{Implications for future research}

Women experiencing postnatal depression were difficult to access. Recruitment of eligible participants was possible but rates were low. In the absence of any routine system for the identification of patients with postnatal depression, critical to any subsequent trial would be a more effective identification strategy, such as screening by health visitors. The present data did not show a significant increase in exercise from this intervention, although the direction of effect from baseline was positive, and self-efficacy for exercise increased significantly.

Given the recent recommendation from NICE that health professionals should consider promoting exercise for the management of postnatal depression, ${ }^{12}$ it is imperative to find out whether this advice is warranted. Evidence from a larger trial is needed on whether, with an effective intervention, it is possible to increase exercise and reduce depression in postnatal women.

\section{Funding body}

This study was funded by the Midlands Research Practice Consortium (MidReC). Richard McManus is supported by a Department of Health Primary Care Postdoctoral Award

\section{Ethical approval}

Ethical approval was obtained from the South Birmingham Local Research Ethics Committee (05/Q2707/96)

\section{Competing interests}

The authors have stated that there are none

\section{Acknowledgements}

We would like to thank the practices, health visitors, and staff at the Mother and Baby Unit, Queen Elizabeth Psychiatric Hospital, Birmingham, who kindly agreed to assist with participant recruitment. We would particularly like to thank those patients who volunteered to participate in the study.

\section{Discuss this article}

Contribute and read comments about this article on the Discussion Forum: http://www.rcgp.org.uk/bjgp-discuss

\section{REFERENCES}

1. Department of Health. Confidential enquiry into maternal and child health: why mothers die 2000-2002. London: Royal College of Obstetricians and Gynaecologists, 2004.

http://www.cemach.org.uk/getdoc/28695c42-0ale-4fd6-8601abd01ad6c162/Saving-Mothers-Lives-Report-2000-2002.aspx (accessed 2 Nov 2007).

2. O'Hara M, Swain A. Rates and risk of postpartum depression - a meta analysis. Int Rev Psychol 1996; 8: 37-54.

3. Gaynes BN, Gavin N, Melzer-Brody S, et al. Perinatal depression: prevalence, screening accuracy and screening outcomes. Summary, Evidence Report/Technology Assessment no.119. Prepared by RTIUniversity of North Carolina Evidence-based Practice Center under contract No.290-02-0016. AHRQ Publication No.05-E006-1. Rockville, MD: Agency for Healthcare Research and Quality, 2005.

4. Murray L. The impact of postnatal depression on infant development. J Child Psychol Psychiatry 1992; 33(3): 543-561.

5. Caplan H, Cogill S, Alexandra H, et al. Maternal depression and the emotional development of the child. Br J Psychiatry 1989; 154: $818-822$

6. Whitton A, Warner R, Appleby L. The pathway to care in postnatal depression: women's attitudes to post-natal depression and its treatment. Br J Gen Pract 1996; 46(408): 427-428.

7. Lawlor DA, Hopker SW. The effectiveness of exercise as an intervention in the management of depression: systematic review and meta-regression analysis of randomized controlled trials. BMJ 2001; 322(7289): 1-8.

8. Stathopoulou G, Powers MB, Berry AC, et al. Exercise interventions for mental health: a quantitative and qualitative review. Clin Psychol 2006; 13(2): 179-193.

9. Daley AJ, MacArthur C, Winter $\mathrm{H}$. The role of exercise as a treatment of postnatal depression: a review. J Midwifery Womens Health 2007; 52(1): 56-62.

10. Armstrong K, Edwards $\mathrm{H}$. The effects of exercise and social support on mothers reporting depressive symptoms: a pilot randomized controlled trial. Int J Mental Health Nurs 2003; 12(2): $130-138$

11. Armstrong K, Edwards $\mathrm{H}$. The effectiveness of a pram-walking exercise programme in reducing depressive symptomatology for postnatal women. Int J Nurs Pract 2004; 10(4): 177-194.

12. National Institute for Health and Clinical Excellence. Antenatal and postnatal mental health. clinical management and service guidance. Reference CG45. London: NICE, 2006.

13. Cox J, Holden J, Sagovsky R. Detection of postnatal depression. Development of the 10-item Edinburgh Postnatal Depression Scale. Br J Psychiatry 1987; 150: 782-786.

14. Godin G, Shephard RJ. A simple method to assess exercise behaviour in the community. Can J Appl Sport Sci 1985; 10(3): 141-146.

15. Marcus BH, Selby VC, Niaura RS. Self-efficacy and the stages of exercise behavior change. Res Q Exerc Sport 1992; 63(1): 60-66.

16. MacArthur C, Winter HR, Bick DE, et al. Effects of redesigned community postnatal care on women's health 4 months after birth: a cluster randomised controlled trial. Lancet 2002; 2(9304): 378-385.

17. Daley AJ, Mutrie, N, Crank H, et al. Exercise therapy in women who have had breast cancer: design of the Sheffield women's exercise and well-being project. Health Educ Res 2004; 19(6): 686-697.

18. Department of Health. At least five a week: evidence on the impact of physical activity and its relationship to health. A report from the Chief Medical Officer. London: Department of Health, 2004. http://www.dh.gov.uk/en/Publicationsandstatistics/Publications/Pu blicationsPolicyAndGuidance/DH_4080994 (accessed 2 Nov 2007).

19. Coyne J, Mitchell AJ. NICE may be discouraging detection of postpartum depression. BMJ 2007; 334(7593): 550. 